\section{Comment on "Linear Wave Dynamics Explains Observations Attributed to Dark Solitons in a Polariton Quantum Fluid"}

In this Comment we challenge the main conclusions of Cilibrizzi and co-workers [1] stating that previous experimental reports on dark solitons in a polariton system [2-6] should be revised as the interpretation in terms of solitons was not sufficiently supported. We show that their analysis on those works is incomplete, as they neglect key evidence of nonlinear behavior. We provide additional analysis of published data, unambiguously confirming the solitonic nature of the observations in Refs. [2-6].

The nonlinear Schrödinger equation [or Gross-Pitaevskii equation, (GPE)] allows for nonlinear localized stable solutions known as dark solitons with the following properties: (i) Solitons appear as oblique dark intensity lines located in the wake of an obstacle. (ii) There is an abrupt jump in the phase of the field across the soliton line. (iii) Analytical expressions relating the phase jump, the width, and depth of the soliton to the fluid velocity and density can be derived from the GPE. (iv) Stable oblique solitons can only appear below a velocity-dependent critical value of the density [7]. (v) Above the critical density, solitons become unstable and are replaced by other behaviors, such as a time-dependent emission of vortices and, at even larger densities, a superfluid behavior.

Even though solitons are intrinsically nonlinear entities, nothing forbids some of their features to present some similarities with observations under suitable conditions in strictly linear systems [8]. An example is reported in Ref. [1], where experiments and simulations with noninteracting polaritons show features that resemble oblique dark solitons, in particular, properties (i) and (ii) listed above. From this, the authors conclude that "Therefore, the previous reports of the observation of dark solitons [2-5] and half-dark solitons [6] which were based on these features have to be reconsidered."
To fairly assess this issue, it is however essential to remind that the claims for the observation of solitons in Refs. [2-6] are not based on the density and phase patterns only, but are further supported by a complete study of the physics as a function of the fluid density [9]. For instance, the observation of a time-dependent regime with a (almost) periodic nucleation of vortex-antivortex pairs, reported in Refs. [3,10,11], is peculiar to nonlinear regimes $[12,13]$.

Furthermore, the solitonic features observed in the nonlinear regime are qualitatively different from the interference patterns reported in the linear regime in Ref. [1], as can be seen in Fig. 1: the interference minima in the linear regime appear in a fanlike shape, and their width rapidly grows after the obstacle [Fig. 1(b)]. On the opposite, the dark solitons in Fig. 1(a) appear as a single pair, they remain tightly focused and their width is related to the fluid density by the characteristic solitonic equations. As the authors of Ref. [1] recognize, this latter criterion is a valid route to unambiguously identify a dark soliton: Fig. 1(d) displays a further analysis of the data shown in Fig. 1(a) [2], clearly confirming that, in contrast to the case of linear interference patterns [Fig. 1(c)], the width of the observed dark intensity lines follows the characteristic behavior of solitons. Equivalent results are obtained when changing the excitation power.

By showing that the observation of dark traces accompanied by sudden phase jumps is not an exclusive feature of soliton physics, Cilibrizzi and coworkers [1] point out the importance of a thorough comparison of linear and nonlinear regimes when analyzing soliton experiments. This is the case of the results published in Refs. [2-6], which indeed satisfy all the above stated criteria for dark soliton physics. On the contrary, criteria (iii)-(v) are not satisfied in experiments performed in the absence of nonlinearities such as the ones of Ref. [1].

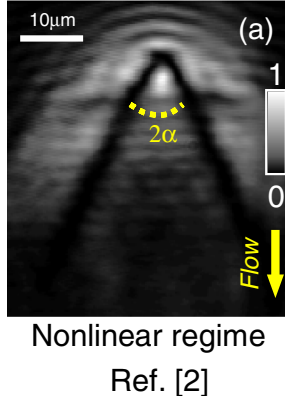

Ref. [2]
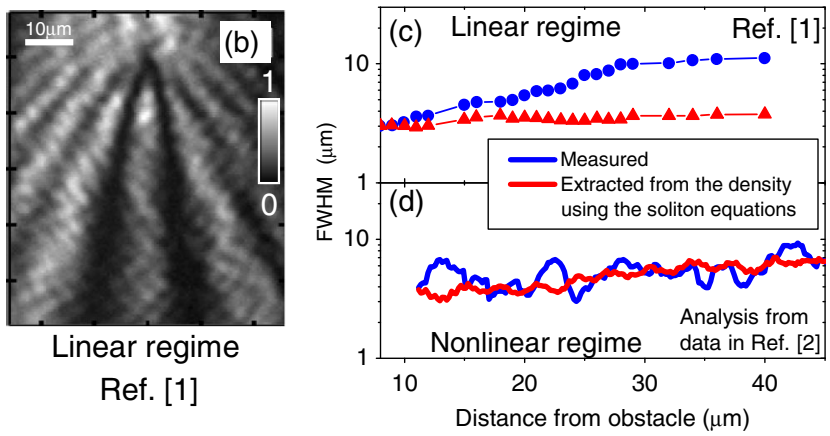

FIG. 1 (color online). (a) Experimentally reported solitons [2] and, (b) interference patterns [1]. (c) Measured full width at half maximum (FWHM) of the density dips in (b), and expected width $w$ from the soliton equations, obtained from the measured local density $n$ using the relation $w=C n^{-1 / 2}$ (with $C$ a fitting parameter); data analysis is in Ref. [1]. (d) Same as (c) for the soliton case reported in (a), following the same criteria to extract the FWHM. 
A. Amo, ${ }^{1}$ J. Bloch, ${ }^{1}$ A. Bramati, ${ }^{2}$ I. Carusotto, ${ }^{3}$ C. Ciuti, ${ }^{4}$

B. Deveaud, ${ }^{5}$ E. Giacobino, ${ }^{2}$ G. Grosso, ${ }^{6}$ A. Kamchatnov, ${ }^{7}$

G. Malpuech, ${ }^{8}$ N. Pavloff, ${ }^{9}$ S. Pigeon, ${ }^{10}$ D. Sanvitto ${ }^{11}$ and

D. D. Solnyshkov 8

${ }^{1}$ Laboratoire de Photonique et Nanostructures LPN/CNRS

Route de Nozay, 91460 Marcoussis, France

${ }^{2}$ Laboratoire Kastler Brossel

Université Pierre et Marie Curie

Ecole Normale Supérieure et CNRS, UPMC Case 74

4 place Jussieu, 75252 Paris Cedex 05, France

${ }^{3}$ INO-CNR BEC Center and Università di Trento 38123 Povo, Italy

${ }^{4}$ Laboratoire Matériaux et Phénomènes Quantiques

Université Paris Diderot-Paris 7 et CNRS

Bâtiment Condorcet, 10 rue Alice Domon et Léonie

Duquet, 75205 Paris Cedex 13, France

${ }^{5}$ Institute of Condensed Matter Physics

École Polytechnique Fédérale de Lausanne (EPFL)

CH-1015 Lausanne, Switzerland

${ }^{6}$ Department of Electrical Engineering and Computer

Science, Massachusetts Institute of Technology

Cambridge, Massachusetts 02139, USA

${ }^{7}$ Institute of Spectroscopy

Russian Academy of Sciences

Moscow, Troitsk 142190, Russia

${ }^{8}$ Institut Pascal, PHOTON-N2, Clermont Université

Blaise Pascal University

CNRS, 24 Avenue des Landais

63177 Aubière Cedex, France

${ }^{9}$ Univ. Paris Sud

CNRS, Laboratoire de Physique Théorique et Modèles

Statistiques, UMR8626, F-91405 Orsay, France

${ }^{10}$ School of Mathematics and Physics

Queen's University Belfast

BT7 1NN Northern Ireland, United Kingdom

${ }^{11}$ CNR NANOTEC - Istituto di Nanotecnologia

Via Monteroni, 73100 Lecce, Italy
Received 23 September 2014; published 17 August 2015

DOI: 10.1103/PhysRevLett.115.089401

PACS numbers: 42.65.Tg, 03.75.Lm, 42.25.Fx, 47.35.Fg

[1] P. Cilibrizzi, H. Ohadi, T. Ostatnicky, A. Askitopoulos, W. Langbein, and P. Lagoudakis, Phys. Rev. Lett. 113, 103901 (2014).

[2] A. Amo, S. Pigeon, D. Sanvitto et al., Science 332, 1167 (2011).

[3] G. Grosso, G. Nardin, F. Morier-Genoud, Y. Léger, and B. Deveaud-Plédran, Phys. Rev. Lett. 107, 245301 (2011).

[4] G. Grosso, G. Nardin, F. Morier-Genoud, Y. Léger, and B. Deveaud-Plédran, Phys. Rev. B 86, 020509 (2012).

[5] B. Deveaud, G. Nardin, G. Grosso et al., in Physics of Quantum Fluids, edited by A. Bramati and M. Modugno (Springer, Berlin Heidelberg, 2013), Vol. 177, p. 99.

[6] R. Hivet, H. Flayac, D. D. Solnyshkov et al., Nat. Phys. 8, 724 (2012).

[7] Note that the finite lifetime of the polaritons is responsible for a sizable shift of the critical density and critical Mach number, as discussed in detail in A. M. Kamchatnov and S. V. Korneev, J. High Energy Phys. 04 (2012) 579 .

[8] A. M. Kamchatnov and N. Pavloff, Eur. Phys. J. D 69, 32 (2015).

[9] A. Amo, J. Bloch, A. Bramati et al., arXiv:1401.7347v1.

[10] G. Nardin, G. Grosso, Y. Leger, B. Piętka, F. MorierGenoud, and B. Deveaud-Plédran, Nat. Phys. 7, 635 (2011).

[11] D. Sanvitto, S. Pigeon, A. Amo et al., Nat. Photonics 5, 610 (2011).

[12] S. Pigeon, I. Carusotto, and C. Ciuti, Phys. Rev. B 83, 144513 (2011).

[13] H. Flayac, D. D. Solnyshkov, and G. Malpuech, Phys. Rev. B 83, 193305 (2011). 